\title{
STANDARD MODULES FOR TABULAR ALGEBRAS
}

\author{
R.M. GREEN \\ Department of Mathematics and Statistics \\ Lancaster University \\ Lancaster LA1 4YF \\ England \\ E-mail: r.m.green@lancaster.ac.uk
}

\begin{abstract}
We introduce cell modules for the tabular algebras defined in a previous work; these modules are analogous to the representations arising from left KazhdanLusztig cells. The standard modules of the title are constructed in an elementary way by suitable tensoring of the cell modules. We show how a certain extended affine Hecke algebra of type $A$ equipped with its Kazhdan-Lusztig basis is an example of a tabular algebra, and verify that in this case our standard modules coincide with other standard modules defined in the literature.
\end{abstract}

\section{To appear in Algebras and Representation Theory}

\section{INTRODUCTION}

In their seminal work [8], Kazhdan and Lusztig showed how left cells in Hecke algebras may be used to construct representations of the algebra. In favourable cases, such as the Hecke algebras associated to the symmetric group, all simple representations may be constructed in terms of these cell representations. Graham and Lehrer [2] developed this idea further by defining cellular algebras in terms of multiplicative properties of a basis. One of the most important results of $[2]$ is a classification of the simple modules of a cellular algebra as explicitly described quotients of the cell modules.

The author thanks Colorado State University for its hospitality during the preparation of part of this paper.

2000 Mathematics Subject Classification. 20C08

Typeset by $\mathcal{A} \mathcal{M} \mathcal{S}-\mathrm{TEX}_{\mathrm{E}}$ 
Tabular algebras were introduced by the author in [4] as a class of associative $\mathbb{Z}\left[v, v^{-1}\right]$-algebras equipped with distinguished bases (tabular bases) and satisfying certain axioms. Although tabular algebras are defined in terms of a seemingly complicated table datum, the main results of $[\mathbf{6}]$ show that, under mild assumptions, this table datum may be recovered up to isomorphism (in a sense made precise in [6]) from the distinguished basis. There are many natural examples of tabular bases given in [4]; these include the natural basis for Brauer's centralizer algebra and various bases arising from Kazhdan-Lusztig type constructions (IC bases).

In this paper, we develop the analogue of cell representations for tabular algebras. The axioms for a tabular algebra mean that these are very easy to define, and that a cell module inherits a nice basis from the corresponding algebra. By suitable tensoring of these cell modules, we define in $\S 1.3$ the standard modules for a tabular algebra. These are constructed in a simple way from the table datum, but their properties may be subtle as we shall explain in $\S 4$.

The left, right and two-sided cells of a tabular algebra may be described purely in terms of the structure constants, just as in the theory of Kazhdan-Lusztig cells. Often a left cell and a right cell will intersect in more than one element; this is not allowed to happen in the case of cellular algebras, but it causes no problems in our construction. Another potential advantage of tabular algebras over cellular algebras is that it is possible to treat some infinite dimensional examples successfully; one of the main results of this paper is Theorem 3.4.5, which shows that a certain extended affine Hecke algebra of type $A$ is an example of a tabular algebra, and that the Kazhdan-Lusztig basis in this case serves as a tabular basis. (This is closely related to N. Xi's results in [17], as we explain in §4.) Using an equivalent construction of standard modules developed in $\S 2$, we show in $\S 4.2$ that the standard modules of the aforementioned Hecke algebra in the sense of tabular algebras agree with the geometrically defined standard modules in the sense of [12].

Some of these ideas have been used implicitly by Graham and Lehrer in the special case of their construction of two-step nilpotent representations of the extended 
affine Hecke algebra of type $A[\mathbf{3}]$. They consider a certain quotient of the extended affine Hecke algebra and classify the simple modules for this quotient in terms of what we call the standard modules. (Both the affine Hecke algebra in question and the quotient considered in $[\mathbf{3}]$ are tabular algebras.) There are also other special cases of this construction in the literature. This suggests some directions for further research; we mention these in the closing remarks.

\section{Cell modules and standard modules for tabular algebras}

We begin in $\S 1$ by recalling the definition of a tabular algebra from [4], and introducing the general concept of standard modules for tabular algebras.

\subsection{Tabular algebras.}

Tabular algebras will be constructed from the normalized table algebras defined below.

Definition 1.1.1. A normalized table algebra is a pair $(\Gamma, B)$, where $\Gamma$ is an associative unital $R$-algebra for some $\mathbb{Z} \leq R \leq \mathbb{C}$ and $B=\left\{b_{i}: i \in I\right\}$ is a distinguished basis for $\Gamma$ such that $1 \in B$, satisfying the following three axioms:

(T1) The structure constants of $\Gamma$ with respect to the basis $B$ lie in $\mathbb{R}^{+}$, the nonnegative real numbers.

(T2) There is an algebra anti-automorphism - of $\Gamma$ whose square is the identity and that has the property that $b_{i} \in B \Rightarrow \overline{b_{i}} \in B$. (We define $\bar{i}$ by the condition $\overline{b_{i}}=b_{\bar{i}}$.)

(T3) Let $\kappa\left(b_{i}, a\right)$ be the coefficient of $b_{i}$ in $a \in \Gamma$. Then

$$
\kappa\left(b_{m}, b_{i} b_{j}\right)=\kappa\left(b_{i}, b_{m} \overline{b_{j}}\right)
$$

for all $i, j, m$.

Notice that the table algebra anti-automorphism is determined by the structure constants: $b_{\bar{i}}$ is the unique basis element with the property that 1 occurs with nonzero coefficient in $b_{i} b_{\bar{i}}$. 
Normalized table algebras as defined above are similar to the table algebras of Arad-Blau [1] and Sunder's discrete hypergroups [16]. Further details may be found in $[4, \S 1.1]$.

The definition of a-function below is due to Lusztig.

Definition 1.1.2. Let $\mathcal{A}$ be the ring of Laurent polynomials $\mathbb{Z}\left[v, v^{-1}\right]$, let $A$ be an $\mathcal{A}$-algebra and let $\mathbf{B}$ be an $\mathcal{A}$-basis of $\mathcal{A}$. For $X, Y, Z \in \mathbf{B}$, we define the structure constants $g_{X, Y, Z} \in \mathcal{A}$ by the formula

$$
X Y=\sum_{Z} g_{X, Y, Z} Z
$$

The a-function is defined by

$$
\mathbf{a}(Z)=\max _{X, Y \in \mathbf{B}} \operatorname{deg}\left(g_{X, Y, Z}\right),
$$

where the degree of a Laurent polynomial is taken to be the highest power of $v$ occurring with nonzero coefficient. We define $\gamma_{X, Y, Z} \in \mathbb{Z}$ to be the coefficient of $v^{\mathbf{a}(Z)}$ in $g_{X, Y, Z}$; this will be zero if the bound is not achieved.

We can now give the definition of a tabular algebra.

Definition 1.1.3. A tabular algebra is an $\mathcal{A}$-algebra $A$, together with a table datum

$$
(\Lambda, \Gamma, B, M, C, *)
$$

satisfying axioms (A1)-(A5) below.

(A1) $\Lambda$ is a finite poset. For each $\lambda \in \Lambda,(\Gamma(\lambda), B(\lambda))$ is a normalized table algebra over $\mathbb{Z}$ and $M(\lambda)$ is a finite set. The map

$$
C: \coprod_{\lambda \in \Lambda}(M(\lambda) \times B(\lambda) \times M(\lambda)) \rightarrow A
$$

is injective with image an $\mathcal{A}$-basis of $A$. We assume that $\operatorname{Im}(C)$ contains a set of mutually orthogonal idempotents $\left\{1_{\varepsilon}: \varepsilon \in \mathcal{E}\right\}$ such that $A=\sum_{\varepsilon, \varepsilon^{\prime} \in \mathcal{E}}\left(1_{\varepsilon} A 1_{\varepsilon^{\prime}}\right)$ and such that for each $X \in \operatorname{Im}(C)$, we have $X=1_{\varepsilon} X 1_{\varepsilon^{\prime}}$ for some $\varepsilon, \varepsilon^{\prime} \in \mathcal{E}$. 
(Typically, the above set of idempotents contains only the identity element of A.) A basis arising in this way is called a tabular basis.

(A2) If $\lambda \in \Lambda, S, T \in M(\lambda)$ and $b \in B(\lambda)$, we write $C(S, b, T)=C_{S, T}^{b} \in A$. Then $*$ is an $\mathcal{A}$-linear involutory anti-automorphism of $A$ such that $\left(C_{S, T}^{b}\right)^{*}=C_{T, S}^{\bar{b}}$, where - is the table algebra anti-automorphism of $(\Gamma(\lambda), B(\lambda))$. If $g \in \mathbb{C}(v) \otimes_{\mathbb{Z}} \Gamma(\lambda)$ is such that $g=\sum_{b_{i} \in B(\lambda)} c_{i} b_{i}$ for some scalars $c_{i}$ (possibly involving $v$ ), we write $C_{S, T}^{g} \in \mathbb{C}(v) \otimes_{\mathcal{A}} A$ as shorthand for $\sum_{b_{i} \in B(\lambda)} c_{i} C_{S, T}^{b_{i}}$. We write $\mathbf{c}_{\lambda}$ for the image under $C$ of $M(\lambda) \times B(\lambda) \times M(\lambda)$; it turns out [4, Proposition 2.3.1] that the a-function is constant on each set $\mathbf{c}_{\lambda}$.

(A3) If $\lambda \in \Lambda, g \in \Gamma(\lambda)$ and $S, T \in M(\lambda)$ then for all $a \in A$ we have

$$
\text { a. } C_{S, T}^{g} \equiv \sum_{S^{\prime} \in M(\lambda)} C_{S^{\prime}, T}^{r_{a}\left(S^{\prime}, S\right) g} \bmod A(<\lambda),
$$

where $r_{a}\left(S^{\prime}, S\right) \in \Gamma(\lambda)\left[v, v^{-1}\right]=\mathcal{A} \otimes_{\mathbb{Z}} \Gamma(\lambda)$ is independent of $T$ and of $g$ and $A(<\lambda)$ is the $\mathcal{A}$-submodule of $A$ generated by the set $\bigcup_{\mu<\lambda} \mathbf{c}_{\mu}$.

(A4) Let $K=C_{S, T}^{b}, K^{\prime}=C_{U, V}^{b^{\prime}}$ and $K^{\prime \prime}=C_{X, Y}^{b^{\prime \prime}}$ lie in $\operatorname{Im}(C)$. Then the maximum bound for $\operatorname{deg}\left(g_{K, K^{\prime}, K^{\prime \prime}}\right)$ in Definition 1.1.2 is achieved if and only if $X=S$, $T=U, Y=V$ and $\kappa\left(b^{\prime \prime}, b b^{\prime}\right) \neq 0$ (where $\kappa$ is as in axiom (T3)). If these conditions all hold and furthermore $b=b^{\prime}=b^{\prime \prime}=1$, we require $\gamma_{K, K^{\prime}, K^{\prime \prime}}=1$.

(A5) There exists an $\mathcal{A}$-linear function $\tau: A \longrightarrow \mathcal{A}$ (the tabular trace), such that $\tau(x)=\tau\left(x^{*}\right)$ for all $x \in A$ and $\tau(x y)=\tau(y x)$ for all $x, y \in A$, that has the property that for every $\lambda \in \Lambda, S, T \in M(\lambda), b \in B(\lambda)$ and $X=C_{S, T}^{b}$, we have

$$
\tau\left(v^{\mathbf{a}(X)} X\right)=\left\{\begin{array}{lll}
1 & \bmod v^{-1} \mathcal{A}^{-} & \text {if } S=T \text { and } b=1, \\
0 & \bmod v^{-1} \mathcal{A}^{-} & \text {otherwise. }
\end{array}\right.
$$

Here, $\mathcal{A}^{-}:=\mathbb{Z}\left[v^{-1}\right]$. We call the elements $C_{S, S}^{1}$ distinguished involutions.

Remark 1.1.4. In [4], a tabular algebra is only required to satisfy axioms (A1)(A3), and an algebra satisfying all five axioms is called a "tabular algebra with trace". However, all the tabular algebras in this paper (and all the most interesting examples) are tabular algebras with trace, so we use the term "tabular algebra" with this narrower meaning. 
Remark 1.1.5. The table datum for a tabular algebra $A$ with tabular basis $\mathbf{B}$ may be reconstructed up to isomorphism (in a sense made precise in [6]) from the structure constants. This means that there is no loss in considering tabular algebras to be pairs $(A, \mathbf{B})$; we shall look at this in more detail in $\S 3$ in the case where $A$ is an affine Hecke algebra and $\mathbf{B}$ is its Kazhdan-Lusztig basis.

\subsection{Cell modules.}

We now introduce cell modules for tabular algebras, the idea of which is implicit in the formulation of axiom (A3). Cell modules are the analogues of the left cell representations in [8] and of the cell modules in [2, Definition 2.1].

Definition 1.2.1. Let $A$ be a tabular algebra with table datum $(\Lambda, \Gamma, B, M, C, *)$. For each $\lambda \in \Lambda$, we define the (left) $A$-module $W(\lambda)$ as follows: $W(\lambda)$ is a free $\mathcal{A}$-module with basis $\left\{C_{S}^{g}: S \in M(\lambda), g \in B(\lambda)\right\}$ and $A$-action defined by

$$
a C_{S}^{g}=\sum_{S^{\prime} \in M(\lambda)} C_{S^{\prime}}^{r_{a}\left(S^{\prime}, S\right) g}
$$

for all $a \in A$. Here, $r_{a}\left(S^{\prime}, S\right)$ is as in axiom (A3), and the notational shorthand is analogous to that in axiom (A3). This is called a (left) cell module for $A$, and the representation it affords is called a (left) cell representation.

Remark 1.2.2. It is clear from axiom (A3) that this module action is well defined. In fact, more is true: the cell modules may be recovered (as modules with distinguished bases) from the tabular basis $\mathbf{B}$ (see Corollary 1.2.6 below).

We could also define right cell modules by applying the tabular anti-automorphism, *.

We now define the left cells of a tabular algebra; this is the one-sided version of the construction in $[\mathbf{4}, \S 3.1]$ referred to in $[\mathbf{4}$, Remark 3.1.2].

Definition 1.2.3. Let $A$ be a tabular algebra. We introduce a relation, $\preceq_{L}$, on the tabular basis by stipulating that $X^{\prime} \preceq_{L} X$ if $X^{\prime}$ appears with nonzero coefficient in $K X$ for some tabular basis element $K$.

The proof of Lemma 1.2.5 will need the following 
Definition 1.2.4. Let $A$ be a tabular algebra with table datum $(\Lambda, \Gamma, B, M, C, *)$. Let $\lambda \in \Lambda$ and $S, T, U, V \in M(\lambda)$. We define $\langle T, U\rangle \in \Gamma(\lambda)\left[v, v^{-1}\right]$ by the condition

$$
C_{S, T}^{1} C_{U, V}^{1} \equiv C_{S, V}^{\langle T, U\rangle} \bmod A(<\lambda) .
$$

If $b \in B(\lambda)$, we define $\langle T, U\rangle_{b} \in \mathcal{A}$ to be the coefficient of $b$ in $\langle T, U\rangle$.

Definition 1.2 .4 is sound by [4, Lemma 2.2.2].

Lemma 1.2.5. Let $A$ be a tabular algebra with table datum $(\Lambda, \Gamma, B, M, C, *)$. Let $\preceq_{L}^{t}$ be the transitive extension of the relation $\preceq_{L}$ of Definition 1.2.3. The relation $\sim_{L}$ on $\operatorname{Im}(C)$ defined by $Y \sim_{L} Z$ if and only if $Y \preceq_{L}^{t} Z$ and $Z \preceq_{L}^{t} Y$ is an equivalence relation. The equivalence classes are known as left cells. Two basis elements $C_{T, U}^{b}$ and $C_{V, W}^{b^{\prime}}$ are in the same left cell if and only if $U=W$ as elements of $\coprod_{\lambda \in \Lambda} M(\lambda)$.

Proof. The proof is a simple adaptation of the proof of [4, Proposition 3.1.3].

The idempotent condition in axiom (A1) shows that $\sim_{L}$ is reflexive. Since $\sim_{L}$ is clearly symmetric and transitive, it is an equivalence relation.

Let $Y=C_{T, U}^{b}$ and $Z=C_{V, U}^{b^{\prime}}$ be basis elements such that $T, U, V \in M(\lambda)$ for the same $\lambda \in \Lambda$; we will show that $Y \sim_{L} Z$. Now

$$
Y^{*} Y=C_{U, T}^{\bar{b}} C_{T, U}^{b} \equiv C_{U, U}^{\bar{b}\langle T, T\rangle b} \bmod A(<\lambda),
$$

which, by [4, Lemma 2.2.3 (i)], contains $C_{U, U}^{1}$ with nonzero coefficient of degree $\mathbf{a}(\lambda)$, where $\mathbf{a}(\lambda)$ is the a-value of any basis element in $\mathbf{c}_{\lambda}$. (The function $\mathbf{a}$ is well defined on $\Lambda$ by [4, Proposition 2.3.1], and the sets $\mathbf{c}_{\lambda}$ are as given in axiom (A2).) There is a similar converse statement: $C_{T, U}^{b} C_{U, U}^{1}$ contains $Y$ with nonzero coefficient. This shows that $Y \sim_{L} C_{U, U}^{1}$. Similarly, we have $Z \sim_{L} C_{U, U}^{1}$ and hence $Y \sim_{L} Z$ as claimed.

Axiom (A3) shows that if $C_{T, U}^{b} \preceq_{L}^{t} C_{V, W}^{b^{\prime}}$, then either $U=W$ or the two basis elements are in different two-sided cells $\mathbf{c}_{\lambda}$. The partial order on $\Lambda$ means that elements in different two-sided cells must be in different left cells. It follows that the equivalence classes are as claimed. 
Corollary 1.2.6. The cell modules (considered as left A-modules with distinguished bases) are determined by the tabular basis.

Proof. By Definition 1.2.3 and Lemma 1.2.5, the left cells depend only on the tabular basis $\mathbf{B}$ and not on the rest of the table datum. Given an left cell $L \subset \mathbf{B}$, we may construct a module with basis $\{l+A(<\lambda): l \in L\}$ with the obvious left $A$-action. This is well defined by the relationship between left cells and two-sided cells mentioned in the last paragraph of the proof of Lemma 1.2.5.

Any given table datum will provide an isomorphism of based $\mathcal{A}$-modules between this module and the module $W(\lambda)$ of Definition 1.2.1: an element $C_{S, T}^{g}$ in $L$ corresponds to the basis element $C_{S}^{g}$.

Remark 1.2.7. The labelling of the basis elements of $W(\lambda)$ is highly dependent on the table datum: for example, there is generally no way to identify which basis elements of $W(\lambda)$ are of the form $C_{S}^{1}$. We do not pursue this, but it may be proved using the results of $[\mathbf{6}]$.

\subsection{Standard modules.}

One of the important properties of the cell modules $W(\lambda)$ is that, as well as being left modules for the tabular algebra $A$, they are also right modules for the hypergroup $\Gamma(\lambda)$. We now show that these actions commute with each other.

The above notation will be fixed throughout $\S 1.3$, as will the table datum for $A$.

Lemma 1.3.1. For any $g \in \Gamma(\lambda)$, the $\mathcal{A}$-linear map $\psi_{g}: W(\lambda) \longrightarrow W(\lambda)$ defined by

$$
\psi_{g}\left(C_{S}^{b}\right):=C_{S}^{b g}
$$

is a homomorphism of left $A$-modules. This gives $W(\lambda)$ the structure of a free right $\Gamma(\lambda)$-module, and the $A$-action and $\Gamma(\lambda)$-action on $W(\lambda)$ commute with each other.

Proof. The map $\psi_{g}$ is a homomorphism by axiom (A3), and the independence of $r_{a}\left(S^{\prime}, S\right)$ from $g$ in that axiom shows that the two actions commute as claimed. Freeness follows from the fact that $C_{S}^{1} \cdot g=C_{S}^{g}$. 
Remark 1.3.2. The $\Gamma(\lambda)$-action defined in Lemma 1.3.1 does in general depend on the table datum chosen. (Contrast this with Corollary 1.2.6.)

Although Lemma 1.3.1 is valid integrally (i.e., over $\mathcal{A}$ ), it will be necessary for some constructions to take the base ring to be a field.

Definition 1.3.3. Let $k$ be a field and let $r \in k^{*}$. Then $k$ is naturally a unital $\mathcal{A}$ module, with $v$ acting as multiplication by $r$. If $W(\lambda)$ is a cell module for a tabular algebra $A$ with table datum $\left(\Lambda, \Gamma, B, M, C\right.$, *), we write $W(\lambda)_{(k, r)}$ for $k \otimes_{\mathcal{A}} W(\lambda)$ and $A_{(k, r)}$ for $k \otimes_{\mathcal{A}} A$, where the $\mathcal{A}$-module structure of $k$ is as above. It is clear that $W(\lambda)_{(k, r)}$ is a left $A_{(k, r)}$-module. Similarly, we write $\Gamma(\lambda)_{(k, r)}$ for $k \otimes_{\mathcal{A}} \mathcal{A} \otimes_{\mathbb{Z}} \Gamma(\lambda)$. Just as in Lemma 1.3.1, $W(\lambda)_{(k, r)}$ is an $A_{(k, r)}-\Gamma(\lambda)_{(k, r)}$-bimodule in which the two actions commute.

Standard modules are defined as follows.

Definition 1.3.4. Maintain the notation of Definition 1.3.3. A standard module for the tabular algebra $A$ is a left $A_{(k, r)}$-module $W(\lambda)_{(k, r)} \otimes_{\Gamma(\lambda)_{(k, r)}} N$, where $N$ is a simple $\Gamma(\lambda)_{(k, r)}$-module.

Remark 1.3.5. The labelling of the standard $A$-modules by pairs $(\lambda, N)$ may depend on the table datum because of Remark 1.3.2, but we shall see that the set of isomorphism classes of standard modules is determined by the tabular basis (Theorem 2.2 .3 (iv)).

\section{Another CONSTRUCTION OF STANDARD MODUles}

In order to develop the properties of standard modules, we give a second construction in terms of the asymptotic tabular algebra. This construction will be valuable in $\S 3$ when we study the affine Hecke algebra of $G L_{n}$ as a tabular algebra. 


\subsection{Asymptotic tabular algebras and bimodules.}

The asymptotic versions of tabular algebras were introduced in [4, §3], using methods from $[\mathbf{1 3}]$.

Definition 2.1.1. Let $A$ be a tabular algebra with trace, and maintain the usual notation. Define $\widehat{X}:=v^{-\mathbf{a}(X)} X$ for any tabular basis element $X \in \operatorname{Im}(C)$. The free $\mathcal{A}^{-}$-module $A_{\lambda}^{-}$is defined to be generated by the elements $\left\{\widehat{X}: X \in \mathbf{c}_{\lambda}\right\}$. We set $t_{X}$ to be the image of $\widehat{X}$ in

$$
A_{\lambda}=A_{\lambda}^{\infty}:=\frac{A_{\lambda}^{-}}{v^{-1} A_{\lambda}^{-}} .
$$

The latter is a $\mathbb{Z}$-algebra with basis $\left\{t_{X}: X \in \mathbf{c}_{\lambda}\right\}$ and structure constants

$$
t_{X} t_{X^{\prime}}=\sum_{X^{\prime \prime} \in \mathbf{c}} \gamma_{X, X^{\prime}, X^{\prime \prime}} t_{X^{\prime \prime}}
$$

where the $\gamma_{X, X^{\prime}, X^{\prime \prime}} \in \mathbb{Z}$ are as in Definition 1.1.2. We also set

$$
A^{\infty}:=\bigoplus_{\lambda \in \Lambda} A_{\lambda}^{\infty}
$$

this is a $\mathbb{Z}$-algebra with basis $\left\{t_{X}: X \in \operatorname{Im}(C)\right\}$ called the asymptotic tabular algebra. We will use the notation $A_{\lambda(k, r)}^{\infty}$ and $A_{(k, r)}^{\infty}$ in the usual way (see Definition 1.3.3) to denote change of scalars by tensoring with $k \otimes_{\mathcal{A}} \mathcal{A} \otimes_{\mathbb{Z}}-$.

We now introduce a certain bimodule that will be helpful in our second construction of standard modules.

Definition 2.1.2. Let $A$ be a tabular algebra (over $\mathcal{A}$ ) with table datum $(\Lambda, \Gamma, B, M, C, *)$. We define $E(\lambda)$ to be the free $\mathcal{A}$-module with basis $\mathbf{c}_{\lambda}$ and we give $E(\lambda)$ the structure of an $A-A_{\lambda}^{\infty}$-bimodule as follows.

The left $A$-module structure of $E(\lambda)$ is the natural one arising from the identification of $E(\lambda)$ with $A(\leq \lambda) / A(<\lambda)$ given by sending a basis element $X \in E(\lambda)$ to $X+A(<\lambda)$. Note that $E(\lambda)$ is isomorphic as an $A$-module to the direct sum of $|M(\lambda)|$ copies of $W(\lambda)$. On the other hand, identifying basis elements of $E(\lambda)$ with basis elements of $A_{\lambda}^{\infty}$ by the correspondence $X \leftrightarrow t_{X}$ defines the right $A_{\lambda}^{\infty}$-action on $E(\lambda)$ via the regular representation over $\mathcal{A}$. 
Lemma 2.1.3. The actions of $A$ and $A_{\lambda}^{\infty}$ on $E(\lambda)$ commute with each other.

Proof. Recall from $\left[\mathbf{4}\right.$, Theorem 3.2.4 (i)] that $A_{\lambda}^{\infty} \cong M_{|M(\lambda)|}(\Gamma(\lambda))$ as $\mathbb{Z}$-algebras; the isomorphism is provided by the table datum. It is thus enough to check that the left $A$-action on $E(\lambda)$ commutes with the action of the element of $A_{\lambda}^{\infty}$ corresponding to $e_{S T} \otimes_{\mathbb{Z}} b$ for $S, T \in M(\lambda)$ and $b \in B(\lambda)$. The latter action is defined by

$$
C_{U, V}^{b^{\prime}} \cdot\left(e_{S T} \otimes_{\mathbb{Z}} b\right)=\delta_{V S} C_{U, T}^{b^{\prime} b}
$$

and axiom (A3) is precisely what is needed for the conclusion to hold.

Since $A_{\lambda}^{\infty}$ is a ring with identity $\left(\Gamma(\lambda)\right.$ has identity and $A_{\lambda}^{\infty}$ is a matrix ring over it) and $E(\lambda)$ affords the regular representation of $A_{\lambda}^{\infty}$, we have $\operatorname{End}_{A_{\lambda}^{\infty}}\left(E_{\lambda}\right) \cong A_{\lambda}^{\infty}$, where the isomorphism is given by left multiplication. Lemma 2.1.3 thus induces a homomorphism $\Phi_{\lambda}: A \longrightarrow \mathcal{A} \otimes_{\mathbb{Z}} A_{\lambda}^{\infty}$ as follows.

Lemma 2.1.4. The homomorphism $\Phi_{\lambda}: A \longrightarrow \mathcal{A} \otimes_{\mathbb{Z}} A_{\lambda}^{\infty}$ induced by Lemma 2.1.3 is given by

$$
\Phi_{\lambda}(X)=\sum_{D \in \mathcal{D}_{\lambda}, Z \in \mathbf{c}_{\lambda}} g_{X, D, Z} t_{Z}
$$

where $\mathcal{D}_{\lambda}:=\left\{C_{S, S}^{1}: S \in M(\lambda)\right\}$.

Proof. We identify $E_{\lambda}$ with $\mathcal{A} \otimes_{Z} A_{\lambda}^{\infty}$ as in Definition 2.1.2. Let $X$ be any basis element of $\operatorname{Im}(C)$ (we do not assume that $X \in \mathbf{c}_{\lambda}$ ). An element $\phi \in \operatorname{End}_{A_{\lambda}^{\infty}}\left(A_{\lambda}^{\infty}\right)$ is defined by the value of $\phi(1)$, so the Lemma will follow once we calculate the value of $X(1)$. Recall that the identity element of $A_{\lambda}^{\infty}$ is $\sum_{D \in \mathcal{D}(\lambda)} t_{D}$; this is Lusztig's property $P_{2}$ in $[4, \S 3.2]$.

Using the left action of $A$ on $E(\lambda)$, we calculate that

$$
X .\left(\sum_{D \in \mathcal{D}_{\lambda}} D\right)=\sum_{D \in \mathcal{D}_{\lambda}, Z \in \mathbf{c}_{\lambda}} g_{X, D, Z} Z
$$

where both sides of the equation are considered as elements of $E(\lambda)$. Considering $E(\lambda)$ as the regular representation of $A_{\lambda}^{\infty}$ again, we see that the endomorphism of 
$E(\lambda)$ induced by $X$ can also be obtained by left multiplication by the element

$$
\sum_{D \in \mathcal{D}_{\lambda}, Z \in \mathbf{c}_{\lambda}} g_{X, D, Z} t_{Z}
$$

of $A_{\lambda}^{\infty}$. The conclusion follows.

The homomorphism $\Phi_{\lambda}$ first appeared in the work of Lusztig; see $[\mathbf{1 3}, \S 1.8]$ and $[\mathbf{4}, \S 3.2]$. It will be useful to have an alternative formula for $\Phi_{\lambda}$ using the notation $\langle T, U\rangle_{b}$ given in Definition 1.2.4. The translation of Lemma 2.1.4 into this notation is as follows.

Lemma 2.1.5. The homomorphism $\Phi_{\lambda}: A \longrightarrow \mathcal{A} \otimes_{\mathbb{Z}} A_{\lambda}^{\infty}$ is given by

$$
\Phi_{\lambda}(a)=\sum_{S, S^{\prime} \in M(\lambda), b \in B(\lambda)}\left(r_{a}\left(S^{\prime}, S\right)\right)_{b} t_{S^{\prime}, S}^{b} .
$$

Here, $t_{S^{\prime}, S}^{b}=e_{S^{\prime}, S} \otimes b$ is the element $t_{Z}$ where $Z=C_{S^{\prime}, S}^{b}$.

\subsection{Constructing $A$-modules using the asymptotic tabular algebra.}

We now show how the cell modules of $\S 1.2$ may be defined starting from the asymptotic tabular algebra.

Definition 2.2.1. Let $A$ be a tabular algebra (over $\mathcal{A}$ ) with table datum

$(\Lambda, \Gamma, B, M, C, *)$. For each $\lambda \in \Lambda$, we define $V(\lambda)$ to be the $\mathbb{Z}$-module with basis $\left\{v_{S}: S \in M(\lambda)\right\}$. The $\mathbb{Z}$-module $W^{\prime}(\lambda):=V(\lambda) \otimes_{\mathbb{Z}} \Gamma(\lambda)$ has the structure of a left $A_{\lambda}^{\infty}$-module via

$$
\left(e_{S T} \otimes b\right) .\left(v_{U} \otimes g\right)=\delta_{T U} v_{S} \otimes b g,
$$

using the isomorphism $A_{\lambda}^{\infty} \cong M_{|M(\lambda)|}(\Gamma(\lambda))$ from [4, Theorem 3.2.4 (i)]. The $\mathcal{A}$ module $\mathcal{A} \otimes_{\mathbb{Z}} W^{\prime}(\lambda)$ becomes an $A$-module using the homomorphism $\Phi_{\lambda}$. If $k$ is a field and $r \in k^{*}$, we write $W^{\prime}(\lambda)_{(k, r)}$ for the $k$-module $k \otimes_{\mathcal{A}} \mathcal{A} \otimes_{\mathbb{Z}} W^{\prime}(\lambda)$; this is a left $A_{(k, r)}$-module in the obvious way.

Proposition 2.2.2. Let $A$ be a tabular algebra (over $\mathcal{A}$ ) with table datum $(\Lambda, \Gamma, B, M, C, *)$, and let $\lambda \in \Lambda$. Let $\theta: W(\lambda) \longrightarrow \mathcal{A} \otimes_{\mathbb{Z}} W^{\prime}(\lambda)$ be the $\mathcal{A}$-module 
isomorphism sending $C_{T}^{b}$ to $1 \otimes v_{T} \otimes b$. Then $\theta$ is an isomorphism of $A-\Gamma(\lambda)$ bimodules.

Proof. Let us first note that the actions of $A$ and $\Gamma(\lambda)$ on $\mathcal{A} \otimes_{\mathbb{Z}} W^{\prime}(\lambda)$ commute. This is because, as one can check, the action of $A_{\lambda}^{\infty}$ on $\mathcal{A} \otimes_{\mathbb{Z}} W^{\prime}(\lambda)$ commutes with the action of $\Gamma(\lambda)$. The same is true for $W(\lambda)$ by Lemma 2.1.3.

It is clear that $\theta$ is compatible with the $\Gamma(\lambda)$ actions on both modules, because $\theta\left(C_{T}^{b g}\right)=v_{T} \otimes b g$ by linearity.

Let $a \in A$. Then using Lemma 2.1.5, we have

$$
\begin{aligned}
a \cdot\left(v_{T} \otimes g\right) & =\Phi_{\lambda}(a) \cdot\left(v_{T} \otimes g\right) \\
& =\left(\sum_{S, S^{\prime} \in M(\lambda), b \in B(\lambda)}\left(r_{a}\left(S^{\prime}, S\right)\right)_{b} t_{S^{\prime}, S}^{b}\right) \cdot\left(v_{T} \otimes g\right) \\
& =\sum_{S^{\prime} \in M(\lambda), b \in B(\lambda)}\left(r_{a}\left(S^{\prime}, T\right)\right)_{b} v_{S^{\prime}} \otimes b g .
\end{aligned}
$$

On the other hand, $a . C_{T}^{g}=\sum_{S^{\prime} \in M(\lambda)} C_{S^{\prime}}^{r_{a}\left(S^{\prime}, T\right) g}$ from the definition of $W(\lambda)$. Because $r_{a}\left(S^{\prime}, T\right)=\sum_{b \in B(\lambda)} r_{a}\left(S^{\prime}, T\right)_{b} b$, we have

$$
\text { a. } C_{T}^{g}=\sum_{S^{\prime} \in M(\lambda)}\left(r_{a}\left(S^{\prime}, T\right)\right)_{b} C_{S^{\prime}}^{b g}
$$

It now follows that $\theta$ is a homomorphism of left $A$-modules.

Theorem 2.2.3. Let $k$ be a field and let $r \in k^{*}$. Let $A$ be a tabular algebra with table datum $(\Lambda, \Gamma, B, M, C, *)$, and let $\lambda \in \Lambda$. Let

$$
\theta_{(k, r)}: W(\lambda)_{(k, r)} \longrightarrow W^{\prime}(\lambda)_{(k, r)}
$$

be the $\mathcal{A}$-module isomorphism sending $C_{S}^{b}$ to $v_{S} \otimes b$. Then

(i) the map $\theta_{(k, r)}$ is an isomorphism of $A_{(k, r)}-\Gamma(\lambda)_{(k, r)}$-bimodules;

(ii) every standard module for $A_{(k, r)}$ is isomorphic to $W^{\prime}(\lambda)_{(k, r)} \otimes_{\Gamma(\lambda)_{(k, r)}} N$ for some simple $\Gamma(\lambda)_{(k, r)}$-module $N$;

(iii) every standard module for $A_{(k, r)}$ is isomorphic to a simple $A_{(k, r)}^{\infty}$-module $N^{\prime}$ regarded as an $A_{(k, r)}$-module by setting a.n $=\Phi_{\lambda}(a)$. $n$ for some $\lambda \in \Lambda$; 
(iv) the isomorphism classes of standard modules for $A_{(k, r)}$ are determined by the tabular basis.

Proof. Part (i) follows from Proposition 2.2.2 after a suitable change of scalars, and part (ii) follows from (i) and Definition 1.3.4.

For (iii), note that the algebras $A_{\lambda(k, r)}$ and $\Gamma(\lambda)_{(k, r)}$ are Morita equivalent, since the first is isomorphic to an $|M(\lambda)|$ by $|M(\lambda)|$ matrix ring over the second. This means that the functor $V(\lambda)_{(k, r)} \otimes_{k} \Gamma(\lambda)_{(k, r)} \otimes_{\Gamma(\lambda)_{(k, r)}}$ - identifies simple $\Gamma(\lambda)_{(k, r)^{-}}$ modules with simple $A_{\lambda(k, r)}$-modules. The conclusion now follows from (ii) once we recall that $A_{\lambda(k, r)}$ is a direct summand of $A_{(k, r)}^{\infty}$.

The definition of standard modules given in (iii) is determined by the tabular basis. At first sight it seems that the definition depends on $\Lambda$, but in fact it only depends on the two-sided cells in $A$, the corresponding homomorphisms $\Phi_{\lambda}$ and the asymptotic algebras $A_{\lambda}^{\infty}$. The maps $\Phi_{\lambda}$ of Lemma 2.1.4 depend only on the tabular basis since the set $\mathcal{D}_{\lambda}$ depends only on the basis (by axiom (A5)). The two-sided cells and asymptotic algebras are determined by the tabular basis by $[\mathbf{6}$, Proposition 2.3.3, Corollary 2.3.4]. These observations prove (iv).

\section{The Affine Hecke Algebra of $G L_{n}$}

In $\S 3$, we show that the asymptotic Hecke algebra of $G L_{n}$ equipped with the Kazhdan-Lusztig basis is a tabular algebra, thus answering in the affirmative a question raised in $[\mathbf{4}, \S 7]$. The proof of this result relies on Xi's solution [17] to Lusztig's conjecture [12, Conjecture 10.5] on the structure of the associated asymptotic Hecke algebra.

\subsection{Hecke algebras.}

We will define Hecke algebras for extended Coxeter groups following the notation of $[17, \S 1.1]$.

Definition 3.1.1. Let $\left(W^{\prime}, S\right)$ be a Coxeter system with $S$ the set of simple reflections. Assume that a commutative group $\Omega$ acts on $\left(W^{\prime}, S\right)$, and consider the 
extended Coxeter group $W=\Omega \ltimes W^{\prime}$. The length function $\ell$ on $W^{\prime}$ and the BruhatChevalley order $\leq$ on $W^{\prime}$ are extended to $W$ by stipulating that $\ell(\omega w):=\ell(w)$, and $\omega w \leq \omega^{\prime} u$ if and only if $\omega=\omega^{\prime}$ and $w \leq u$, where $\omega, \omega^{\prime}$ are in $\Omega$ and $w, u$ are in $W^{\prime}$.

The Hecke algebra $\mathcal{H}$ of $(W, S)$ over $\mathcal{A}$ with parameter $v^{2}$ is an associative algebra over $\mathcal{A}$, with free $\mathcal{A}$-basis $\left\{T_{w}: w \in W\right\}$ and defining relations

$$
\begin{aligned}
\left(T_{s}-v^{2}\right)\left(T_{s}+1\right) & =0 \text { if } s \in S \\
T_{w} T_{u} & =T_{w u} \text { if } \ell(w u)=\ell(w)+\ell(u) .
\end{aligned}
$$

Let $^{-}: \mathcal{A} \longrightarrow \mathcal{A}$ be the $\mathbb{Z}$-linear ring homomorphism $\mathcal{A}$ defined by $\bar{v}=v^{-1}$. Then we have a bar involution of $\mathcal{H}$ defined by

$$
\overline{\sum a_{w} T_{w}}=\sum \bar{a}_{w} T_{w^{-1}}^{-1}, \quad a_{w} \in \mathcal{A} .
$$

For each $w \in W$ there is a unique element $C_{w}$ in $\mathcal{H}$ such that $\overline{C_{w}}=C_{w}$ and

$$
C_{w}=v^{-\ell(w)} \sum_{y \leq w} P_{y, w}\left(v^{2}\right) T_{y},
$$

where $P_{y, w}$ is a polynomial in $v$ of degree $\leq \frac{1}{2}(\ell(w)-\ell(y)-1)$ if $\ell(w)>\ell(y)$ and $P_{w, w}=1$.

The basis $\left\{C_{w}: w \in W\right\}$ is called the Kazhdan-Lusztig basis of the Hecke algebra $H$ and the polynomials $P_{y, w}$ are the Kazhdan-Lusztig polynomials.

We can now introduce the affine Hecke algebra of $G L_{n}$. Xi calls this the "extended affine Hecke algebra associated to $S L_{n}(\mathbb{C})$ " [17, §8.4], and Graham-Lehrer [3] call it the "extended affine Hecke algebra of type $A$ ".

Definition 3.1.2. The affine Hecke algebra of $G L_{n}$ arises from the construction in Definition 3.1 .1 by setting $\left(W^{\prime}, S\right)$ to be the Coxeter system of type $\widehat{A}_{n-1}$ (for $n \geq 3)$ and $\Omega$ to be the cyclic group $\mathbb{Z}_{n}$ acting by rotations of the Coxeter graph.

Remark 3.1.3. The Kazhdan-Lusztig basis arising from the extended Coxeter group $(W, S)$ is closely related to that arising from the Coxeter group $\left(W^{\prime}, S\right)$. The basis 
elements of the larger Hecke algebra are precisely those of the form $T_{\omega} C_{u}$, where $\omega \in$ $\Omega$ and $u \in W^{\prime}$; this follows from the characterization of the basis given in Definition 3.1.1 because $T_{\omega}$ is invariant under the involution. In particular, $C_{\omega}=T_{\omega}$. It also follows easily from Definition 3.1.1 that $T_{\omega} C_{u} T_{\omega^{-1}}$ is a Kazhdan-Lusztig basis element, namely $C_{\omega u \omega^{-1}}$ (consider the leading term $T_{u}$ of $C_{u}$ ). These properties of the basis are well known; see for example the proof of [17, Proposition 1.4.6 (b)].

Remark 3.1.4. We note that there are other quite different presentations of the algebra given in Definition 3.1.2, but the set-up given is convenient for studying Kazhdan-Lusztig theory.

The following standard result will be useful later.

Lemma 3.1.5. Let $(W, S)$ be an extended Coxeter group. The $\mathcal{A}$ linear map * on $\mathcal{H}$ that sends $T_{w}$ to $T_{w^{-1}}$ is an algebra anti-automorphism, and we have $C_{w}^{*}=C_{w^{-1}}$ for all $w \in W$.

Proof. The first assertion comes from symmetry properties of the relations for $\mathcal{H}$ given in Definition 3.1.1 and the fact that $\ell(w)=\ell\left(w^{-1}\right)$ for all $w \in W$. Applying * to the defining expression for $C_{w}$ in Definition 3.1.1 shows that $C_{w}^{*}$ satisfies the required degree bounds.

It only remains to show that $\overline{C_{w}^{*}}=C_{w}^{*}$. It follows from the definition of $\mathcal{H}$ that it is generated as an $\mathcal{A}$-algebra by $\left\{C_{s}: s \in S\right\}$ and $\left\{C_{\omega}: \omega \in \Omega\right\}$ and it is enough to prove that $*$ and $^{-}$commute on $\mathcal{H}$. This follows because $* 0^{-}$and ${ }^{-} \circ *$ are both $\mathcal{A}$-antilinear ring homomorphisms fixing the algebra generating set just given.

We now turn our attention to a particularly important set of involutions in the Coxeter group $W^{\prime}$.

Definition 3.1.6. Let $\left(W^{\prime}, S\right)$ be a Coxeter group. Following Lusztig [10, $\left.§ 1.3\right]$, we find that $\mathbf{a}(z) \leq \ell(z)-2 \operatorname{deg} P_{e, z}$, and we define the set of distinguished involutions of $W^{\prime}$ to be

$$
\mathcal{D}=\left\{z \in W^{\prime}: 2 \operatorname{deg} P_{e, z}=\ell(z)-\mathbf{a}(z)\right\}
$$


It is not immediately clear that the distinguished involutions are involutions, but this is proved in [10, Proposition 1.4]. The terminology of Definition 3.1.6 will eventually be seen to be compatible with the distinguished involutions mentioned in axiom (A5) for a tabular algebra.

\subsection{Kazhdan-Lusztig cells.}

For the rest of $\S 3$, we shall usually denote the affine Hecke algebra of $G L_{n}$ by $A$, the Kazhdan-Lusztig basis by $\mathbf{B}$ and the associated extended Coxeter group by $W$. We aim to show that $\mathbf{B}$ is a tabular basis for $A$. The table datum for $(A, \mathbf{B})$ will be given in terms of the two-sided Kazhdan-Lusztig cells of the Hecke algebra. These may be defined in terms similar to Definition 1.2.3.

Definition 3.2.1. Let $\left(W^{\prime}, S\right)$ be a Coxeter group and let $x, w \in W^{\prime}$. We write $x \leq_{L} w$ if there is a chain

$$
x=x_{0}, x_{1}, \ldots, x_{r}=w,
$$

possibly with $r=0$, such that for each $i<r, C_{x_{i}}$ occurs with nonzero coefficient in the linear expansion of $C_{s} C_{x_{i+1}}$ for some $s \in S$ such that $s x_{i+1}>x_{i+1}$.

The preorder $\leq_{R}$ on $W^{\prime}$ is defined by the condition $x \leq_{R} w \Leftrightarrow x^{-1} \leq_{L} w^{-1}$, and the preorder $\leq_{L R}$ is that generated by $\leq_{L}$ and $\leq_{R}$.

If $W=\Omega \ltimes W^{\prime}$ is an extended Coxeter group, $w, u \in W^{\prime}$ and $\omega, \omega^{\prime}$ in $\Omega$, we say that $\omega w \leq_{L} \omega^{\prime} u$ (respectively, $\omega w \leq_{R} \omega^{\prime} u, \omega w \leq_{L R} \omega^{\prime} u$ ) if $w \leq_{L} u$ (respectively $\left.w \leq_{R} u, w \leq_{L R} u\right)$.

The transitive preorder $\leq_{L}$ yields an equivalence relation $\sim_{L}$ on $W$ or $W^{\prime}$ (where $x \sim_{L} w$ if and only if $x \leq_{L} w$ and $\left.w \leq_{L} x\right)$ whose equivalence classes are called the left cells of $W$. The preorders $\leq_{R}$ and $\leq_{L R}$ yield equivalence relations $\sim_{R}$ and $\sim_{L R}$ on $W$ whose equivalence classes are called right cells and two-sided cells, respectively. Note that the left (respectively right, two-sided) cells are partially ordered by $\leq_{L}$ (respectively $\left.\leq_{R}, \leq_{L R}\right)$.

The above definition agrees with the original definition in [8] for the Coxeter group $W^{\prime}$; see [7, Remark 1.2.3] for an explanation. 
The next result is immediate from the definitions.

Lemma 3.2.2. Let $(W, S)$ be an extended Coxeter group corresponding to the Coxeter group $\left(W^{\prime}, S\right)$. The two-sided (respectively left, right) cells of $W$ are precisely the subsets of the form $\{\omega c: \omega \in \Omega, c \in \mathbf{c}\}$, where $\mathbf{c}$ is a two-sided (respectively left, right) cell of $W^{\prime}$.

The following well known result will be useful in connection with tabular bases.

Lemma 3.2.3. Let $(W, S)$ be an extended Coxeter group with Hecke algebra $\mathcal{H}$ as defined in §3.1. For any $\xi, \xi^{\prime} \in \mathcal{H}$ and $w \in W$ we write

$$
\xi C_{w} \xi^{\prime}=\sum_{z \in W} h_{z} C_{z}
$$

with $h_{z} \in \mathcal{A}$. Then $h_{z}=0$ unless $z \leq_{L R} w$. If $\xi^{\prime}=1$ (respectively, $\xi=1$ ), we have $h_{z}=0$ unless $z \leq_{L} w$ (respectively, $z \leq_{R} w$ ). Furthermore, the preorders $\leq_{L}, \leq_{R}$ and $\leq_{L R}$ are characterized by these conditions.

Proof. The last assertion follows from Definition 3.2.1, because $C_{s} C_{w}=\left(v+v^{-1}\right) C_{w}$ if $s, w \in W^{\prime}$ and $s w<w$ (where $W^{\prime}$ is the Coxeter group corresponding to $W$ ). The other assertions were proved in $[\mathbf{9}, \S 4.3]$ in the case where $W$ is a Coxeter group. For the general case, we need only check the case where the elements $\xi$ and $\xi^{\prime}$ are of the form $T_{\omega}$ for $\omega \in \Omega$, and in this case the result is immediate from Definition 3.2 .1 .

For the rest of this section, let $\left(W^{\prime}, S\right)$ be a Coxeter group of type $\widehat{A}_{n-1}$ and $(W, S)$ be the extended Coxeter group described in Definition 3.1.2.

In this case, the cells have a nice description established by Shi [14], who showed that the two-sided cells are labelled by the partitions of $n$. This description is given by a map, $\sigma$, from $W^{\prime}$ to the set of partitions of $n$, whose fibres are the two-sided cells. (The reader is referred to $[\mathbf{1 4}]$ for the definition of $\sigma$, which we do not require here.) We extend $\sigma$ to a map from $W$ to the partitions of $n$ given by $\sigma(\omega w):=\sigma(w)$ for $\omega \in \Omega$. 
The partitions of $n$ are naturally partially ordered by the dominance order: if $\lambda$ and $\mu$ are two partitions of $n$, we say that $\lambda \unrhd \mu(\lambda$ dominates $\mu)$ if for all $k$ we have

$$
\sum_{i=1}^{k} \lambda_{i} \geq \sum_{i=1}^{k} \mu_{i}
$$

Theorem 3.2.4 (Shi). Let $(W, S)$ be the extended Coxeter group $\Omega \ltimes W^{\prime}$ of Definition 3.1.2 corresponding to the affine Hecke algebra of $G L_{n}$, and let $y, w \in W$. Then $y \leq_{L R} w$ in the sense of Kazhdan-Lusztig if and only if $\sigma(y) \unrhd \sigma(w)$. In particular, $y$ and $w$ are in the same two-sided cell (i.e., $y \leq_{L R} w \leq_{L R} y$ ) if and only if $\sigma(y)=\sigma(w)$.

Proof. The case where $y, w \in W^{\prime}$ is dealt with in $[\mathbf{1 5}, \S 2.9]$, and the general case is immediate from Lemma 3.2.2.

The relevance of Theorem 3.2.4 for our purposes is that the partitions of $n$ partially ordered by dominance will form the poset $\Lambda$ of axiom (A1) for a tabular algebra.

\subsection{The asymptotic affine Hecke algebra of $G L_{n}$.}

Throughout $\S 3.3$, we shall consider the algebra $(A, \mathbf{B})$, where $A$ is the affine Hecke algebra of $G L_{n}$ from Definition 3.1.2 and $\mathbf{B}$ is its Kazhdan-Lusztig basis. We shall denote the Coxeter group and the extended Coxeter group corresponding to $(A, \mathbf{B})$ by $\left(W^{\prime}, S\right)$ and $(W, S)$ respectively.

The Hecke algebra has an asymptotic algebra that arises from a construction analogous to that of Definition 2.1.1. We recall the definition from $[\mathbf{1 7}, \S 1.5]$.

Definition 3.3.1. Let $(A, \mathbf{B})$ be the affine Hecke algebra of $G L_{n}$ equipped with the Kazhdan-Lusztig basis. Define the structure constants $g_{x, y, z}$ by the formula

$$
C_{x} C_{y}=\sum_{z \in W} g_{x, y, z} C_{z}
$$

and define the corresponding integers $\gamma_{x, y, z}$ as in Definition 1.1.2. The asymptotic affine Hecke algebra of $G L_{n}, J$, is the free $\mathbb{Z}$-algebra with basis $\left\{t_{w}: w \in W\right\}$ and 
structure constants

$$
t_{x} t_{y}=\sum_{z \in W} \gamma_{x, y, z} t_{z}
$$

If $\mathbf{c}$ is a two-sided cell in $W$, we define the ring $J_{\mathbf{c}}$ to be the $\mathbb{Z}$-module with free basis $\left\{t_{w}: w \in \mathbf{c}\right\}$.

Lemma 3.3.2. The algebra $J$ of Definition 3.3.1 decomposes as a direct sum of two-sided ideals $J \cong \bigoplus_{\mathbf{c}} J_{\mathbf{c}}$, where the sum ranges over all two-sided cells of $W$.

Proof. This is a consequence of the theory of cells in affine Weyl groups developed in $[\mathbf{1 0}]$; see $[\mathbf{1 7}, \S 1.5]$ for further discussion of this property.

Definition 3.3.3. A based ring is a pair $(R, B)$, where $R$ is a unital $\mathbb{Z}$-algebra with free $\mathbb{Z}$-basis $B$ and nonnegative structure constants. An isomorphism of based rings is an isomorphism abstract $\mathbb{Z}$-algebras compatible with the distinguished bases.

The asymptotic Hecke algebra $J$ is an example of a based ring; the positivity property follows from results in $[\mathbf{9}, \S 3]$ (see $[\mathbf{1 7}, \S 1.3]$ for the case of extended Coxeter groups).

The structure of the asymptotic algebra of Definition 3.3.1 as a based ring was established by $\mathrm{Xi}$ in [17], thus verifying a conjecture of Lusztig [12, Conjecture 10.5] in a the case of type $A$. Lemma 3.3.2 quickly reduces this problem to that of understanding the algebras $J_{\mathbf{c}}$.

Theorem 3.3.4 (Xi). Let $\mathbf{c}$ be the two-sided cell associated by the map $\sigma$ of Theorem 3.2.4 to the partition $\lambda$ of $n$. Then $J_{\mathbf{c}}$ is isomorphic as a based ring to the full matrix ring $M_{n_{\mu}}(\Gamma(\lambda))$ over a certain (commutative) based ring $\Gamma(\lambda)$. Here, $n_{\mu}=n ! /\left(\mu_{1} ! \cdots \mu_{r} !\right)$, where $\mu$ is the dual partition of $\lambda$.

Proof. This follows from [17, Theorem 2.3.2].

Proposition 3.3.5. Let $\lambda$ be a partition of n, let $\mathbf{c}$ be the two-sided cell corresponding to $\lambda$ as in Theorem 3.2.4 and let $L$ be a left cell contained in $\mathbf{c}$. Let $J_{L \cap L^{-1}}$ be the $\mathbb{Z}$-module with basis $\left\{t_{w}: w \in L \cap L^{-1}\right\}$. Then $J_{L \cap L^{-1}}$ is a subring of $J$ 
isomorphic (as a based ring) to the based ring $\Gamma(\lambda)$ of Theorem 3.3.4. Furthermore, these based rings are normalized table algebras over $\mathbb{Z}$, and the table algebra anti-automorphism of $J_{L \cap L^{-1}}$ is induced by the map $t_{w} \mapsto t_{w^{-1}}$.

Proof. It follows from [10, Theorem 1.10] and Lemma 3.2.2 that $L$ contains a unique distinguished involution, $d$, in the sense of Definition 3.1.6. It follows easily from Definition 3.2.1 that $L^{-1}$ is a right cell; it also contains $d$ since $d^{2}=1$.

It can be shown using standard properties of asymptotic Hecke algebras that $J_{L \cap L^{-1}}$ is a subring of $J$ (see $[\mathbf{1 7}, \S 1.5]$ ), and this subring is isomorphic as a based ring to $\Gamma(\lambda)$ by $\left[\mathbf{1 7}\right.$, Theorem 2.3.2]. We now show that $J_{L \cap L^{-1}}$ is a normalized table algebra.

The identity element is $t_{d}$, which follows from the properties of the integers $\gamma$ developed in $[\mathbf{1 0}]$; see $[\mathbf{1 7}, \S 1.5]$ for remarks on the extended Coxeter group case.

Axiom (T1) follows from the positivity properties of $[\mathbf{9}, \S 3]$; the structure constants are integers by definition.

The map ${ }^{-}: t_{w} \mapsto t_{w^{-1}}$ clearly leaves the set $L \cap L^{-1}$ stable and permutes the basis of $\Gamma(\lambda)$. Since $\mathbf{a}(z)=\mathbf{a}\left(z^{-1}\right)$ by $[\mathbf{9}$, Proposition 2.2], we can apply the map * of Lemma 3.1.5 to a product $C_{x} C_{y}$ to show that that $g_{x, y, z}=g_{y^{-1}, x^{-1}, z^{-1}}$ and $\gamma_{x, y, z}=\gamma_{y^{-1}, x^{-1}, z^{-1}}$, which proves that ${ }^{-}$is an anti-automorphism satisfying axiom (T2). (Compare with $[\mathbf{1 0}, 1.1$ (f)], which proves the above identity for $\gamma$ in the case of Coxeter groups.)

Another standard property of $\gamma$ that follows from the results of $[\mathbf{1 0}]$ is $\gamma_{x, y, z}=$ $\gamma_{y, z^{-1}, x^{-1}}($ see $[\mathbf{1 7}, \S 1.3(\mathrm{~b})])$. Combining this with the property in the previous paragraph, we find that $\gamma_{x, y, z}=\gamma_{z, y^{-1}, x}$. This is precisely the property needed to prove axiom (T3).

\subsection{Tabular structure of the affine Hecke algebra of $G L_{n}$.}

We continue to concentrate on $(A, \mathbf{B})$, the affine Hecke algebra of $G L_{n}$ (equipped with the Kazhdan-Lusztig basis), and on its extended Coxeter group $(W, S)$. To prove axiom (A3), we need to take a closer look at the structure constants arising 
from the basis $\mathbf{B}$.

Definitions 3.4.1 and 3.4.2 appear in $[\mathbf{1 3}, \S 1]$.

Definition 3.4.1. Let $(A, \mathbf{B})$ be as above, and let $\mathbf{c}$ be a two-sided KazhdanLusztig cell. We define $A_{\mathbf{c}}$ to be the free $\mathcal{A}$-module with $\mathbf{c}$ as a basis. This has the structure of an $A-A$ bimodule: the left module structure is given by the formula

$$
b . b^{\prime}=\sum_{b^{\prime \prime} \in \mathbf{c}} g_{b, b^{\prime}, b^{\prime \prime}} b^{\prime \prime}
$$

where $b \in \mathbf{B}$ and $b^{\prime} \in \mathbf{c}$, and the right module structure is given by the same formula but with $b \in \mathbf{c}$ and $b^{\prime} \in \mathbf{B}$. The two module structures commute by associativity of $A$ and the partial order on the cells.

We now introduce a second indeterminate, $v^{\prime}$. We denote by $A^{\prime}$ the $\mathbb{Z}\left[v^{\prime}, v^{\prime-1}\right]$ algebra obtained from $A$ by substituting $v^{\prime}$ for $v$. We write $g_{b, b^{\prime}, b^{\prime \prime}}(v)$ for $g_{b, b^{\prime}, b^{\prime \prime}}$ to emphasize that $g_{b, b^{\prime}, b^{\prime \prime}} \in \mathcal{A}$.

\section{Definition 3.4.2.}

Maintain the above notation. Let $G_{\mathbf{c}}$ be the free $\mathbb{Z}\left[v, v^{-1}, v^{\prime}, v^{\prime-1}\right]$-module with basis $\mathbf{c}$. We endow $G_{\mathbf{c}}$ with the structure of a left $A$-module using the formula in Definition 3.4.1. We also endow $G_{\mathbf{c}}$ with the structure of a right $A^{\prime}$-module, also using the formula in Definition 3.4.1 but substituting $g_{b, b^{\prime}, b^{\prime \prime}}\left(v^{\prime}\right)$ for $g_{b, b^{\prime}, b^{\prime \prime}}(v)$.

Proposition 3.4.3. The module $G_{\mathbf{c}}$ of Definition 3.4.2 is an $A-A^{\prime}$ bimodule, i.e., the two module structures commute.

Proof. It is enough to check that expressions of the form $b \cdot b^{\prime} \cdot b^{\prime \prime}$ denote well defined elements of $G_{\mathbf{c}}$ whenever $b$ is a basis element of $A, b^{\prime \prime}$ is a basis element of $A^{\prime}$ and $b^{\prime}$ is a basis element of $G_{\mathbf{c}}$. Let $w, w^{\prime}, w^{\prime \prime}$ denote the elements of $W$ indexing the basis elements $b, b^{\prime}, b^{\prime \prime}$ respectively.

Remark 3.1.3 reduces the problem to the consideration of the case where $w, w^{\prime}$, $w^{\prime \prime}$ all lie in the Coxeter group $W^{\prime}$, because computation of the product $C_{\omega} C_{u}$ does not involve $v$ when $\omega \in \Omega$. The proof is completed by [13, Theorem 2.2]. 
Proposition 3.4.4. Maintain the notation of Definition 3.4.1. For any $b_{1}, b_{2}, b_{3}$, $\beta^{\prime} \in \mathbf{B}$ such that $\beta^{\prime} \in \mathbf{c}$ and $b_{2} \in \mathbf{c}$, we have

$$
\sum_{\beta \in \mathbf{c}} g_{b_{1}, b_{2}, \beta}(v) \gamma_{\beta, b_{3}, \beta^{\prime}}=\sum_{\beta \in \mathbf{c}} g_{b_{1}, \beta, \beta^{\prime}}(v) \gamma_{b_{2}, b_{3}, \beta}
$$

Proof. This is formally the same as [13, Proposition 1.9 (a)]. The proof is valid in this context due to Proposition 3.4.3 and the fact (proved in [9, Theorem 5.4]) that the a-function is constant on elements of $\mathbf{c}$.

We now have all the ingredients to prove the main result.

Theorem 3.4.5. The Kazhdan-Lusztig basis is a tabular basis for the affine Hecke algebra of $G L_{n}(n \geq 3)$, and the distinguished involutions of the tabular basis agree with the distinguished involutions in the sense of Lusztig.

Proof. Let $\Lambda$ be the set of partitions of $n$, partially ordered by dominance so that $\lambda \leq \mu$ means $\lambda \unrhd \mu$. For each $\lambda \in \Lambda$, let $M(\lambda)$ be the set of numbers from 1 up to $n_{\mu}$, where $n_{\mu}$ is as in Theorem 3.3.4, and let $(\Gamma(\lambda), B(\lambda))$ be the normalized table algebra of Proposition 3.3.5. The map $C$ of the table datum takes a triple $\left(m, b, m^{\prime}\right)$ from $M(\lambda) \times B(\lambda) \times M(\lambda)$ and associates to it the Kazhdan-Lusztig basis element $C_{w}$ (where $w \in W$ ) for which $t_{w}$ corresponds to the element $e_{m m^{\prime}} \otimes b$ under the isomorphism of Theorem 3.3.4. The map $*$ sends $C_{w}$ to $C_{w^{-1}}$.

We now check the axioms of Definition 1.1.3.

Axiom (A1) follows easily once we notice that the identity element of the Hecke algebra is a Kazhdan-Lusztig basis element.

We saw in Lemma 3.1.5 that $*$ is an anti-automorphism. We now need to check that it is compatible with the map $C$ of the table datum. Fix $\lambda \in \Lambda$, let $\mathbf{c}$ be the corresponding two-sided cell (as in Theorem 3.2.4) and let ${ }^{-}$denote the table algebra anti-automorphism of $\Gamma(\lambda)$ as in Proposition 3.3.5. We saw in the proof of that proposition that the map $t_{w} \mapsto t_{w^{-1}}$ extends to an algebra anti-automorphism of $J$. It follows from Theorem 3.3.4, Proposition 3.3.5 and [17, Lemma 2.3.1] that 
this anti-automorphism acts on $J_{\mathbf{c}}$ by sending $e_{m m^{\prime}} \otimes b$ to $e_{m^{\prime} m} \otimes \bar{b}$, where ${ }^{-}$is the table algebra anti-automorphism of $\Gamma(\lambda)$. Axiom (A2) now follows.

The claims about the partial order in axiom (A3) follow from Lemma 3.2.3 and Theorem 3.2.4. For the other claims, we note that the left $A$-module $A_{\mathbf{c}}$ of Definition 3.4.1 is a right $J_{\mathbf{c}}$-module affording the regular representation after the two bases are identified in the obvious way. Proposition 3.4.4 says that these two actions commute with each other. Now label each basis element of $A_{\mathbf{c}}$ by an element $e_{m m^{\prime}} \otimes b$ as given by the isomorphism of Theorem 3.3.4, and the remaining claims are consequences of Proposition 3.4.4.

Axiom (A4) follows easily from Theorem 3.3.4, which allows the elements $\gamma_{K, K^{\prime}, K^{\prime \prime}}$ to be computed. The axiom says that

$$
\left(e_{m_{1} m_{2}} \otimes b_{1}\right)\left(e_{m_{3} m_{4}} \otimes b_{2}\right)
$$

contains the basis element $e_{m_{5} m_{6}} \otimes b_{3}$ with nonzero coefficient if and only if both factors come from the same summand $J_{\mathbf{c}}, m_{5}=m_{1}, m_{2}=m_{3}, m_{6}=m_{4}$ and $b_{3}$ occurs with nonzero coefficient in $b_{1} b_{2}$. The assertion regarding $\gamma_{K, K^{\prime}, K^{\prime \prime}}=1$ is also clear.

For axiom (A5), we define an $\mathcal{A}$-linear function $\tau$ on the Hecke algebra $\mathcal{H}$ of $(W, S)$ by the condition

$$
\tau\left(\sum a_{w} T_{w}\right):=a_{1}
$$

We claim $\tau(x y)=\tau(y x)$ for all $x, y \in \mathcal{H}$; it is enough to check that this holds when $x=T_{s}$ or $x=T_{\omega}$ for $\omega \in \Omega$, and this follows easily from the relations in $\S 3.1$. (The corresponding result for Coxeter groups is $[\mathbf{9}, 1.4 .1]$.) Since $*$ sends $T_{w}$ to $T_{w^{-1}}$, it is clear that $\tau(x)=\tau\left(x^{*}\right)$.

To complete the proof, let us denote by $\left(W^{\prime}, S\right)$ the Coxeter group corresponding to $(W, S)$. If $z \in W^{\prime}$, it follows from Definition 3.1.6 and the formula for $C_{z}$ in Definition 3.1.1 that

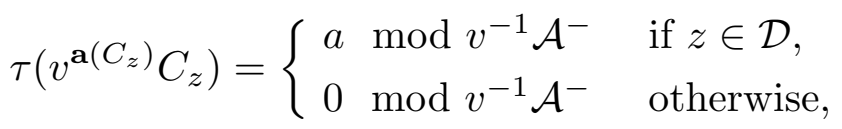


where $a \in \mathbb{Z}$. By $[\mathbf{1 0}$, Proposition $1.4(\mathrm{a})]$, we see that $P_{e, z}$ is monic when $z \in \mathcal{D}$, and this proves that $a=1$.

Now if $u \in \Omega$, Remark 3.1.3 shows that $\tau\left(C_{u z}\right)$ is zero unless $u$ is the identity, and we have already dealt with the latter case. We conclude that

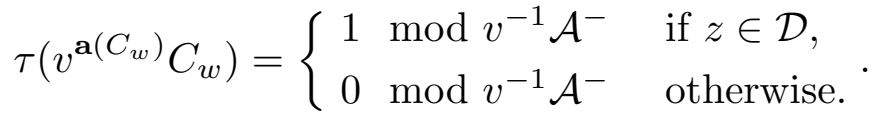

It remains to show that the distinguished involutions in the sense of Definition 3.1.6 coincide with the distinguished involutions of axiom (A5). As mentioned in $[\mathbf{1 7}, \S 1.5]$, the ring $J$ has an identity, namely $\sum_{d \in \mathcal{D}} t_{d}$. From the construction in $[4$, Lemma 3.2.2], we now see that

$$
\left\{C_{d}: d \in \mathcal{D}\right\}=\left\{C_{S, S}^{1}: S \in M(\lambda), \lambda \in \Lambda\right\}
$$

where the $C$ on the right is as in the definition of table datum. This means that the two senses of the term "distinguished involution" agree in this case, and the proof is complete.

\section{Applications}

In $\S 4$, we compare the results of this paper with some related results in the literature.

\subsection{Description of Kazhdan-Lusztig cells using the table datum.}

It is not hard to show that the cell modules for the affine Hecke algebra of $G L_{n}$ as a tabular algebra are compatible with the cell representations in the sense of Kazhdan-Lusztig; we do this now for the sake of easy reference.

Proposition 4.1.1. Let $A$ be the affine Hecke algebra of $G L_{n}(n \geq 3)$, let $\mathbf{B}$ be its Kazhdan-Lusztig basis, and let $(W, S)$ be the corresponding Coxeter system. Let $(\Lambda, \Gamma, B, M, C, *)$ be the table datum for $(A, \mathbf{B})$ given in the proof of Theorem 3.4.5. Let $x, y \in W$, and write $C_{T, U}^{b}=C_{x}$ and $C_{V, W}^{b^{\prime}}=C_{y}$, where $C_{x}, C_{y} \in \mathbf{B}$, $T, U \in M(\lambda)$ and $V, W \in M\left(\lambda^{\prime}\right)$ for some $\lambda, \lambda^{\prime} \in \Lambda$. Then: 
(i) $x \sim_{L R} y \Leftrightarrow \lambda=\lambda^{\prime}$;

(ii) $x \sim_{L} y \Leftrightarrow \lambda=\lambda^{\prime}$ and $U=W$;

(iii) $x \sim_{R} y \Leftrightarrow \lambda=\lambda^{\prime}$ and $T=V$.

Proof. We first prove (ii). Two elements $C_{S, T}^{b}$ and $C_{U, V}^{b^{\prime}}$ are in the same left cell of $\mathbf{B}$ (in the sense of tabular algebras given in Lemma 1.2.5) if and only if $\lambda=\lambda^{\prime}$ and $U=W$. The preorder generating this equivalence relation is given in Definition 1.2.3, and the latter is clearly compatible with the characterization of the preorder $\leq_{L}$ given in Lemma 3.2.3. The proof of (ii) now follows from the definition of left cells in the Kazhdan-Lusztig sense.

Part (iii) follows from (ii) by applying the tabular anti-automorphism $*$. This sends $C_{S, T}^{b}$ to $C_{T, S}^{\bar{b}}$ by definition, and sends $C_{w}$ to $C_{w^{-1}}$ by Lemma 3.1 .5 ; recall from Definition 3.2.1 that inversion sends left cells to right cells (in the sense of Kazhdan-Lusztig).

The proof of (i) is similar to the proof of (ii), replacing Lemma 1.2 .5 by [4, Proposition 3.1.3], Definition 1.2.3 by [4, Definition 3.1.1] and using the characterization of $\leq_{L R}$ in Lemma 3.2.3.

Remark 4.1.2. Although there are many possible table data for the pair $(A, \mathbf{B})$, Proposition 4.1.1 can be shown to be independent of the choice of table datum using ideas from $[\mathbf{6}]$.

One application of Proposition 4.1.1 is that it shows that, in the situation under consideration, the cell modules of $\$ 1.2$ afford the left cell representations defined in $[8]$.

\subsection{Standard modules for affine Hecke algebras.}

Combining Theorem 2.2.3 with Theorem 3.4.5 gives a construction of a set of "standard modules" for the affine Hecke algebra of $G L_{n}$. (Notice that, by Definition 1.3.4 and Theorem 2.2.3 (ii), these modules can be defined in terms of the representation theory of the table algebras $\Gamma(\lambda)$ without reference to the asymptotic algebra.) The next result justifies the use of the term "standard modules" 
introduced in $\S 1.3$.

Proposition 4.2.1. Let $A$ be the affine Hecke algebra of $G L_{n}(n \geq 3)$, let $\mathbf{B}$ be its Kazhdan-Lusztig basis, and let $(W, S)$ be the corresponding Coxeter system. The (isomorphism classes of) standard modules associated to the pair $(A, \mathbf{B})$ in the sense of $\S 1.3$ agree with the standard modules $\mathcal{K}_{u, s, \rho}$ in the sense of Lusztig [12].

Note. Recall from Theorem 2.2.3 (iv) that the isomorphism classes of standard modules for a tabular algebra depend only on the basis.

Proof. The modules $\mathcal{K}_{u, s, \rho}$ are defined in [12], and are called "standard modules" in the introduction to [12]. By [12, Theorem 4.2], each module $\mathcal{K}_{u, s, \rho}$ is isomorphic to precisely one module ${ }^{\Phi} E$, where $E$ is a simple $J$-module made into an $\mathcal{H}$-module by applying a certain homomorphism $\Phi: \mathcal{H} \longrightarrow J$. (The set-up in [12] is that the base ring is a field, $k$, and the parameter $v$ acts by scalar multiplication by $r \in k^{*}$, as in our $\S 2.2$.) The map $\Phi$ is defined to be $\bigoplus_{\lambda \in \Lambda} \Phi_{\lambda}$, where $\Phi_{\lambda}$ is as in Lemma 2.1.4 and $\Lambda$ is as in the proof of Theorem 3.4.5; this is a restatement of the formula in $[\mathbf{1 2}, \S 1.4]$ using the identifications of Theorem 3.4.5 and Proposition 4.1.1. Since $A_{\lambda}^{\infty}$ (i.e., $J_{\mathbf{c}}$ for some two-sided cell c) is a direct summand of $J$ and $E$ is simple,

${ }^{\Phi} E$ is isomorphic to ${ }^{\Phi_{\lambda}} E$ for some $\lambda \in \Lambda$. The conclusion follows from Theorem 2.2 .3 (iii).

Remark 4.2.2. An interesting problem is the determination of the decomposition matrix of the standard modules into simple modules. If the scalar $r$ (as in the proof above) is not a root of unity or is equal to $1,[\mathbf{1 1}$, Theorem 3.4] shows that the standard modules are simple, but at a root of unity the situation is much more complicated.

\subsection{Relationship with two-step nilpotent representations.}

We now outline how we can use the language of tabular algebras to set up some of the main results in Graham and Lehrer's work on two-step nilpotent representations of the Hecke algebra of $G L_{n}[\mathbf{3}]$. 
As usual, we let $A$ be the affine Hecke algebra of $G L_{n}(n \geq 3)$, let $\mathbf{B}$ be its Kazhdan-Lusztig basis, and let $(W, S)$ be the corresponding Coxeter system. It follows (for example from [5, Theorem 3.4]) that if we consider the set

$$
\Lambda_{c}:=\left\{\lambda \in \Lambda: \lambda_{1} \geq 3\right\}
$$

of all partitions of $n$ whose first part exceeds 3 , then the set of basis elements $\bigcup_{\lambda \in \Lambda_{c}} \mathbf{c}_{\lambda}$ spans an ideal of $A$. Quotienting $A$ by this ideal yields an algebra denoted by $\widetilde{T L}_{n}^{a}(q)$ in $[\mathbf{3},(1.7)]$. It is an easy consequence of Theorem 3.4 .5 that this algebra is tabular with tabular basis given by the nonzero images of the elements $C_{w}$. This basis is closely related to, but not always the same as, the basis used in [3]. Either basis may be handled using only combinatorics, which is not the case for the full Kazhdan-Lusztig basis B.

The "cell modules" of $[\mathbf{3}]$ are simply the standard modules of $\widetilde{T L}_{n}^{a}(q)$ with the projection of the Kazhdan-Lusztig basis as the tabular basis. (The construction of these modules is rather similar to our Definition 1.3.4.) One of the main achievements of $[\mathbf{3}]$ is the determination of the decomposition matrix of the standard modules into simples. It turns out (see, for example, [3, Theorem 5.5 (ii)]) that each standard module has a unique simple quotient, and every simple module is isomorphic to one arising in this way. This behaviour is familiar from the theory of cellular algebras in [2].

\subsection{Concluding remarks.}

As well as proving Lusztig's conjecture for the affine Hecke algebra of $G L_{n}$, Xi also considers the case where $\Omega$ is the group $\mathbb{Z}$, also acting by rotations of the Coxeter graph. The conclusion (given in $[\mathbf{1 7}, \S 8.2]$ ) is that the conjecture holds, and we have an analogue of Theorem 3.3.4. A version of Theorem 3.4 .5 will also hold, with some light modifications to the proof.

Xi's main results in [17] may be paraphrased in the language of tabular algebras by saying that they verify that the table data of certain extended affine Hecke algebras are as predicted by Lusztig; this is a highly nontrivial result. An independent 
proof of Theorem 3.4.5 would achieve some of the steps required in the proofs of the main results of $[\mathbf{1 7}, \S 8.4]$.

As regards standard modules, it would be very interesting to know whether the behaviour described in $§ 4.3$ is typical; that is, can one always construct a set of simple modules from the heads of the standard modules? One problem to be solved here is a replacement for the bilinear form used in [2] or [3] to construct the simple modules from the standard modules, especially in the case where the table algebras involved are noncommutative.

\section{REFERENCES}

[1] Z. Arad and H.I. Blau, On Table Algebras and Applications to Finite Group Theory, J. Algebra 138 (1991), 137-185.

[2] J.J. Graham and G.I. Lehrer, Cellular algebras, Invent. Math. 123 (1996), 1-34.

[3] J.J. Graham and G.I. Lehrer, The two-step nilpotent representations of the extended affine Hecke algebra of type A, Compositio Math. (to appear).

[4] R.M. Green, Tabular algebras and their asymptotic versions, J. Algebra 252 (2002), 27-64.

[5] R.M. Green, On 321-avoiding permutations in affine Weyl groups, J. Algebraic Combin. 15 (2002), 241-252.

[6] R.M. Green, Categories arising from tabular algebras, Glasgow Math. J. (to appear; math.QA/0207097).

[7] R.M. Green and J. Losonczy, Fully commutative Kazhdan-Lusztig cells, Ann. Inst. Fourier (Grenoble) $5 \mathbf{1}$ (2001), 1025-1045.

[8] D. Kazhdan and G. Lusztig, Representations of Coxeter groups and Hecke algebras, Invent. Math. 53 (1979), 165-184.

[9] G. Lusztig, Cells in affine Weyl groups, Algebraic groups and related topics, Adv. Studies Pure Math 6, North-Holland and Kinokuniya, Tokyo and Amsterdam, 1985, pp. 255-287.

[10] G. Lusztig, Cells in affine Weyl groups, II, J. Alg. 109 (1987), 536-548.

[11] G. Lusztig, Cells in affine Weyl groups, III, J. Fac. Sci. Tokyo U. (IA) 34 (1987), $223-243$.

[12] G. Lusztig, Cells in affine Weyl groups, IV, J. Fac. Sci, Tokyo U. (IA) 36 (1989), 297-328.

[13] G. Lusztig, Quantum groups at $v=\infty$, Prog. Math. 131 (1995), 199-221.

[14] J.Y. Shi, The Kazhdan-Lusztig cells in certain affine Weyl groups, Lecture Notes in Mathematics 1179 (1986), Spinger, Berlin.

[15] J.Y. Shi, The partial order on two-sided cells of certain affine Weyl groups, J. Alg. 176 (1996), 607-621.

[16] V.S. Sunder, $I I_{1}$ factors, their bimodules and hypergroups, Trans. Amer. Math. Soc. 330 (1992), 227-256.

[17] N. Xi, The based ring of two-sided cells of affine Weyl groups of type $\tilde{A}_{n-1}$, Mem. Amer. Math. Soc. 157 (2002), no. 749. 